\title{
Supernumerary chromosomes in Nephrops norvegicus L. (Crustacea, Decapoda)
}

\author{
A. M. DEIANA*, E. COLUCCIA, A. MILIA $+\&$ S. SALVADORI \\ Dipartimento di Biologia Animale ed Ecologia and †Dipartimento di Biologia Sperimentale, Universita' di Cagliari, \\ Viale Poetto 1, 09126 Cagliari, Italy
}

\begin{abstract}
Supernumerary chromosomes have been identified in the chromosome complement of Nephrops norvegicus L. by C-banding and restriction endonuclease-induced banding. These chromosomes showed peculiar features of typical B chromosomes in terms of heterochromatin content, asynapsis and distorted segregation. The presence of these chromosomes can, at least partially, account for the numerical variability already observed in this species; $2 n$ and $n$ values determined by counting these chromosomes separately gave a less variable number of chromosomes in the complement. Q-banding was useful to characterize some large B chromosomes with intercalary and subtelomeric bands as well as entirely positive short arms in some small submetacentric chromosomes. Nucleolar organizer regions have been identified in four pairs of chromosomes by silver staining.
\end{abstract}

Keywords: B chromosomes, chromosome banding, Nephrops norvegicus.

\section{Introduction}

A remarkable intraspecific numerical variability of the chromosome complements has been documented in Nephropidae (for reviews, see Nakamura, 1988; Corni et al., 1989).

The large number, small size and peculiar structure of the chromosomes present in the species belonging to the family make it difficult to define their karyotype. However, it seems unlikely that the variability observed among different individuals (Roberts, 1969; Hughes, 1982; Corni, 1989) is merely the result of a counting bias. The presence of supernumerary chromosomes and their peculiar behaviour could better explain these variations.

In the present study on Nephrops norvegicus, we provide evidence of the presence of chromosomes with some of the features and characteristic behaviour of B chromosomes (Jones \& Rees, 1982a,b; Jones, 1985). C-banding and DdeI-endonuclease digestion proved to be a useful tool in allowing a sharp differentiation of the chromosomes, a clear identification of meiotic figures and a better understanding of the nature of chromosome variability in this species.

*Correspondence.

\section{Materials and methods}

Specimens were collected by trawling off the Sardinian coast at a depth of 200-700 m. Chromosome preparations from the testicular tissue of 10 males were made using the Doussau de Bazignan and Ozouf-Costaz technique (1985) and from embryos according to a modified Klinkhardt technique (1992). Briefly, ova with embryos in the first stage were incubated in 0.02 per cent colchicine in sea water for 3-5 $\mathrm{h}$ and then in a hypotonic solution of $0.075 \mathrm{M} \mathrm{KCl}$ for $60 \mathrm{~min}$. The hypotonic medium was twice replaced by fixative (3:1 methanol/acetic acid) for $20 \mathrm{~min}$ and once more overnight. The chorion and the yolk were removed and the cells were dispersed in a drop of acetic acid (40 per cent) and spread over the slide surface. Embryo metaphases were not used in determining chromosome number because of the high loss of chromosomes resulting from hypotonic treatment.

\section{Staining}

Slides were stained in 25 per cent Wright's stain (in $0.06 \mathrm{M}$ phosphate buffer, $\mathrm{pH}$ 6.8) for $8 \mathrm{~min}$, briefly rinsed in distilled water and air-dried. Wright's stain stock solution was prepared as follows: $0.25 \mathrm{~g}$ of 
Wright's stain (Sigma) dissolved in $100 \mathrm{~mL}$ of methyl alcohol, stirred for $1 \mathrm{~h}$, filtered and stored in an oven at $37^{\circ} \mathrm{C}$.

\section{C-banding}

Fixed chromosomes, aged 6-14 days, were treated with 5 per cent $\mathrm{Ba}(\mathrm{OH})_{2} .8 \mathrm{H}_{2} \mathrm{O}$ for $3-5 \mathrm{~min}$ at $50^{\circ} \mathrm{C}$, briefly rinsed in $0.1 \mathrm{~N} \mathrm{HCl}$ followed by a rinse in distilled water, incubated in $2 \times \mathrm{SSC}$ for $1 \mathrm{~h}$ at $60^{\circ} \mathrm{C}$ and stained with 25 per cent Wright's stain for 7 $\min$.

\section{NOR silver staining and restriction endonuclease banding}

These techniques were performed according to Cau et al. (1988).

\section{Fluorescence microscopy}

Q-banding was obtained following Casperson et al. (1969).

\section{Results}

\section{Mitotic chromosomes}

The chromosome complement of $N$. norvegicus comprises metacentric, submetacentric and acrocentric chromosomes and shows a great variability of the $2 n$ and $n$ values, within and among individuals (Farmer, 1974; Corni et al., 1989). A few metacentric and submetacentric chromosomes, often without homologues, are remarkably larger than all the others (Fig. 1a).

All centromeric regions showed C-positive reaction (Fig. 1c,d). Moreover, entirely C-positive chromosomes, variable in number within and among individuals, were observed. Similar results were obtained with the restriction endonuclease DdeI with sharper resolution (Figs 2 and 3).

Quinacrine identified some AT-rich clusters localized on the short arms of some small submetacentric chromosomes. Moreover, some of the large chromosomes showed intercalary and subtelomeric bands on both arms (Fig. 4a).

Ag-staining revealed four to eight nucleoli in the interkinetic nucleus of the embryos (Fig. 4e). In mitotic metaphases, five to eight Ag-positive regions were identified, localized in the short arms of three pairs of small acrocentric chromosomes as well as in the centromeric region of a small pair of metacentric chromosomes (Fig. 4d).

\section{Meiotic chromosomes}

In metaphase I the bivalents, showing a very regular, circular arrangement were dumbbell-, ring- or crossshaped; some highly condensed, chromatin bodies were identified (Fig. 1b). C-banding and DdeI staining showed the presence of some asynaptic chromosomes; they appeared tightly condensed and strongly stained, the primary constriction being often difficult to identify (Figs 1d, $2 \mathrm{~b}$ and $3 \mathrm{~b}$ ). They also presented distorted segregation. In fact, in metaphases II from the same meiocyte it was possible to observe that these chromosomes are distributed randomly between the daughter cells (Figs $2 c-e$ and $3 c-e$ ). On account of their peculiar structure and behaviour, these chromosomes could be defined as B chromosomes whereas 'A chromosomes' indicates all the other members of the complement.

\section{Numerical variability}

The diploid values in spermatogonial metaphases ranged from 131 to 140 . Variability was present among different individuals as well as among cells of the same individual (Tables 1 and 2). In metaphase I, counting of bivalents after conventional staining was of ambiguous interpretation because the asynaptic B chromosomes could not be easily distinguished from bivalents. After $\mathrm{C}$ and DdeI treatment, which facilitated the identification of asynaptic chromosomes, we obtained a modal number of 64 bivalents and $8 \mathrm{~B}$ chromosomes in metaphase I. Congruent values were obtained in metaphase II for the $A$ chromosomes whereas the number of $\mathrm{B}$ chromosomes ranged from three to five.

\section{Morphology of B chromosomes}

According to their different features three groups of B chromosomes were identified.

1 The first group corresponds to the largest chromosome of the complement which is only partially heterochromatic; after banding it was easily identifiable because of large, positive paracentromeric blocks of chromatin (Fig. 2a). In meiotic metaphase I, it was asynaptic and doubled up, often forming a univalent ring. For this reason, it could be regarded as an isochromosome. In some specimens this chromosome was without homologues, both in the spermatogonial and in the meiotic metaphases (Fig. $2 \mathrm{a}-\mathrm{e})$. In other cases two homologues were distinguishable which were asynaptic, doubled up in meiotic metaphases and showed distorted segregation (Fig. 3a-e). 


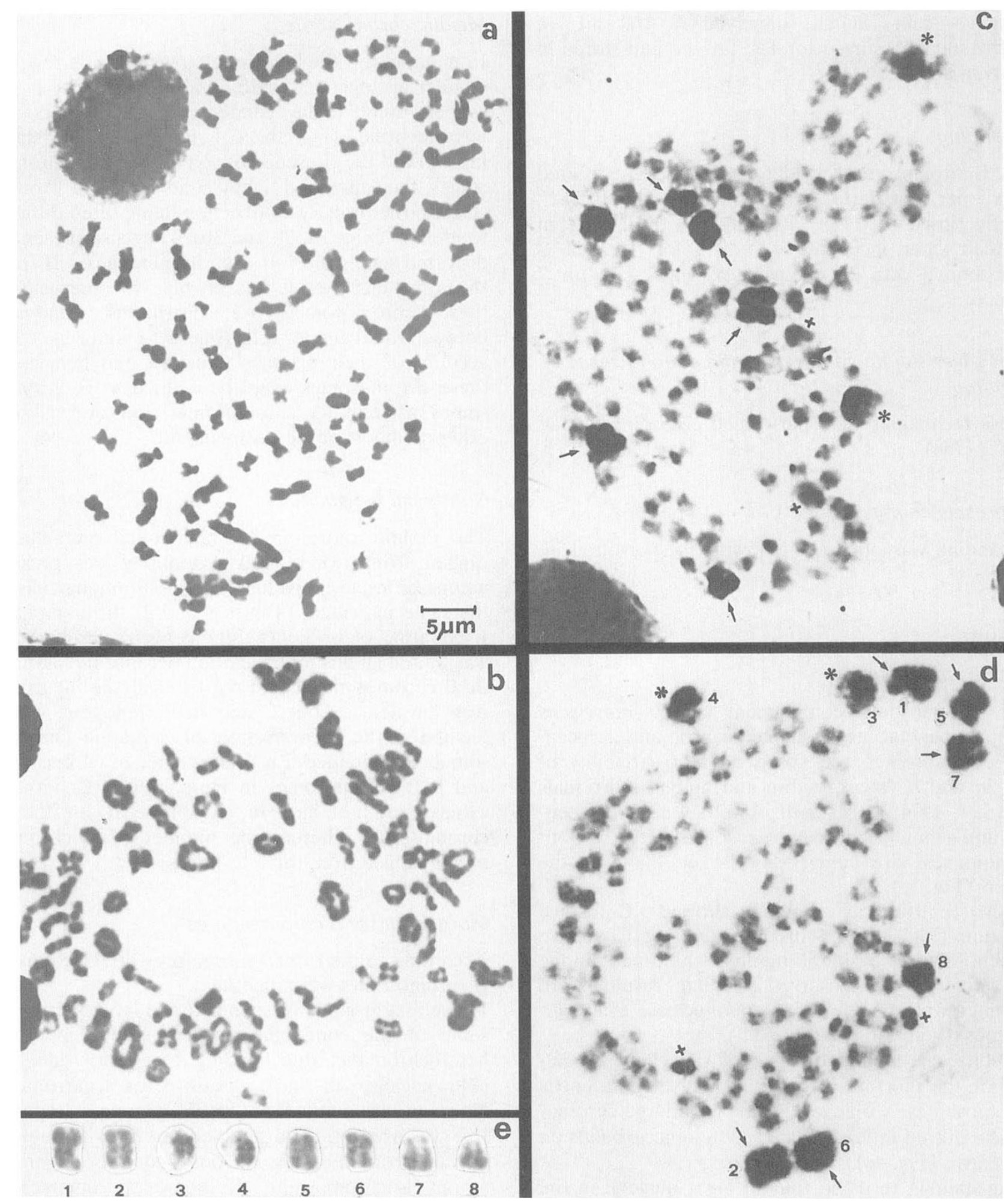

Fig. 1 (a) Embryonal metaphase and (b) meiotic metaphase I after Wright's staining. B chromosomes are indistinguishable from the other members of the complement. (c) Spermatogonial metaphase and (d) meiotic metaphase I after C-banding; the centromeric regions of all chromosomes and the whole B chromosomes are C-positive. The identifiable B chromosomes are: (1) a pair of large chromosomes with an extensive, paracentromeric positive region $\left({ }^{*}\right) ;(2)$ large metacentric, submetacentric and acrocentric chromosomes (arrowed); (3) small chromosomes, mainly acrocentric $(+)$. In (e), the large B chromosomes of (d) show their univalent nature after a shorter photographic exposure. 


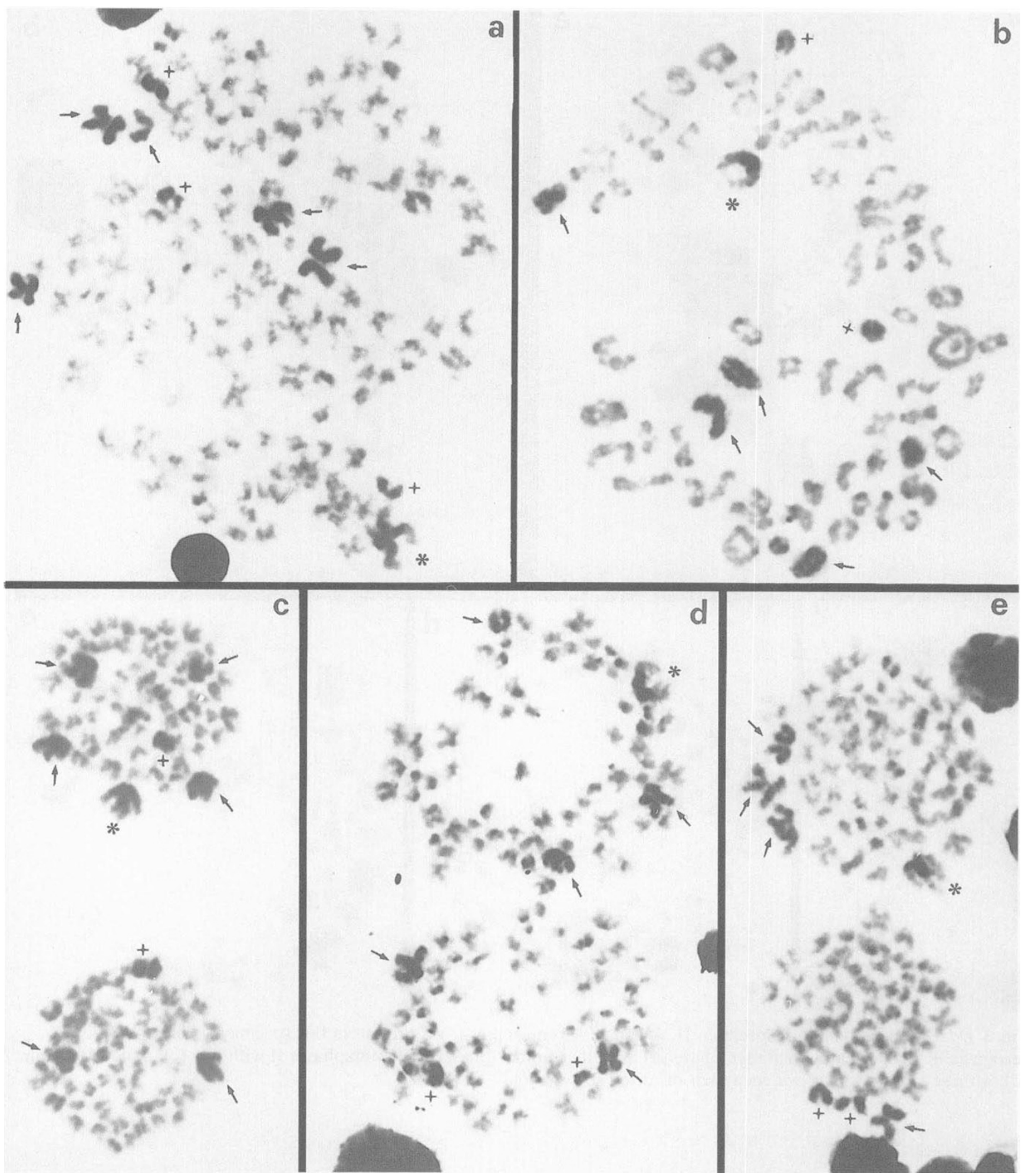

Fig. 2 DdeI-induced banding. Specimen I. (a) Spermatogonial mitosis; the identifiable B chromosomes are: (1) a single, large chromosome with an extensive, paracentromeric positive region $\left({ }^{*}\right) ;(2)$ large metacentric, submetacentric and acrocentric chromosomes (arrowed); (3) small chromosomes, mainly acrocentric (+). (b) Meiotic metaphase I. B chromosomes are recognizable as they are strongly stained and asynaptic. The large chromosome $\left(^{*}\right)$ makes a peculiar univalent ring. (c-e) Pairs of two metaphases II; each of them comes from the same meiocyte I; it is evident that B chromosomes undergo a distorted segregation. 


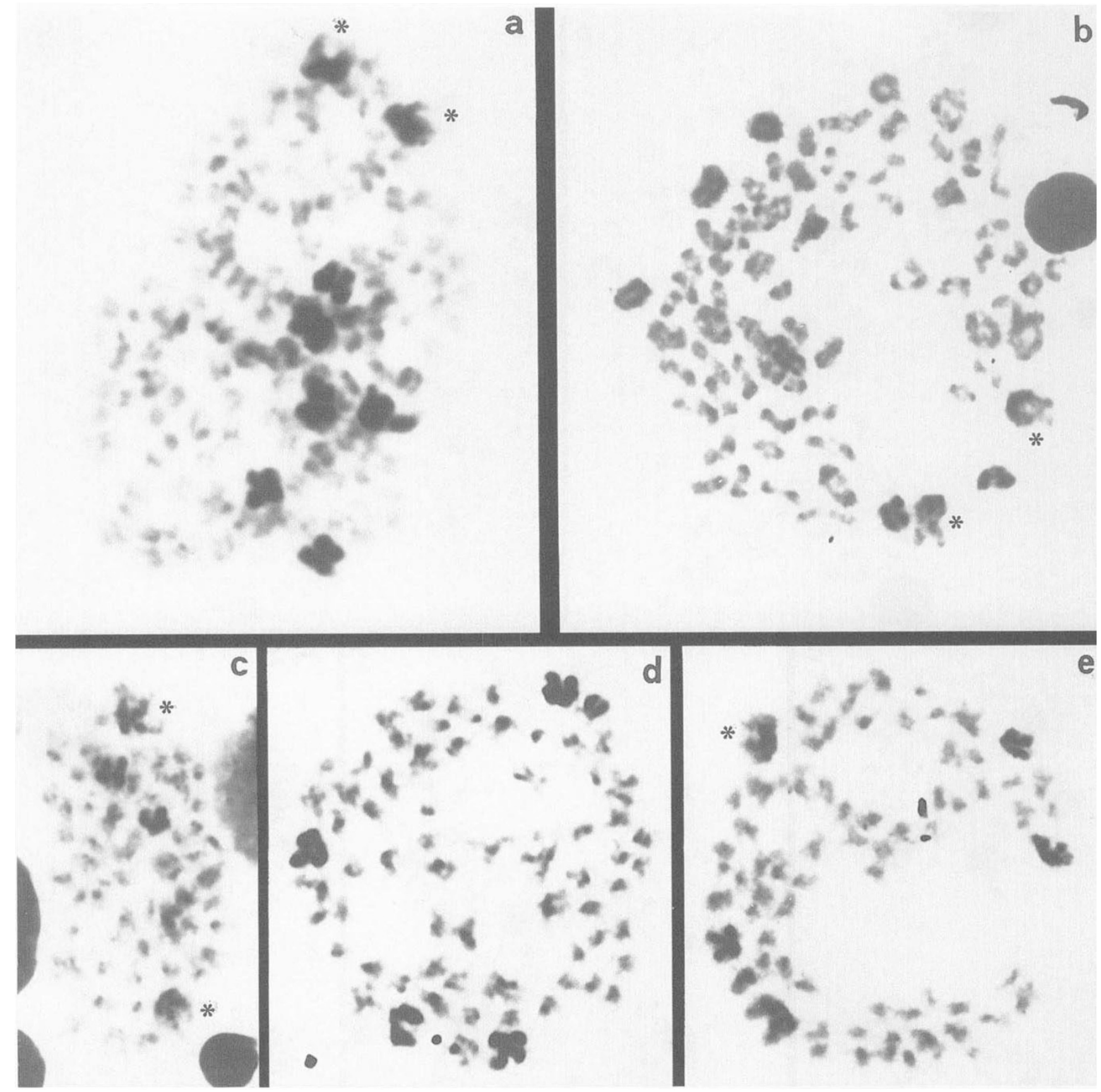

Fig. 3 DdeI-induced banding. Specimen II. A pair of chromosomes $\left(^{*}\right)$ is present in this specimen. They are easily recognizable in spermatogonial metaphase (a) as well as in metaphase I (b). Metaphases II with two (c), none (d) or one (e) of these chromosomes evidence their distorted segregation.

2 The second group comprises completely heterochromatic, large B chromosomes including one/two acrocentric chromosomes and a few (maximum five) metacentric and submetacentric chromosomes (Fig. 2a). In meiosis they appeared so condensed as to be indistinguishable from each other (Figs $1 d, 2 b$ and 3b). These chromosomes appear randomly distrib- uted in metaphase II (Figs $2 c-e$ and $3 c-e$ ). In this group, the eventual presence of sex-linked chromosomes could not be ruled out.

3 The third group is composed of very small-sized, heterochromatic chromosomes, variable in number (four to five the most; Figs 1c,d and 2a).

(c) The Genetical Society of Great Britain, Heredity, 76, 92-99. 

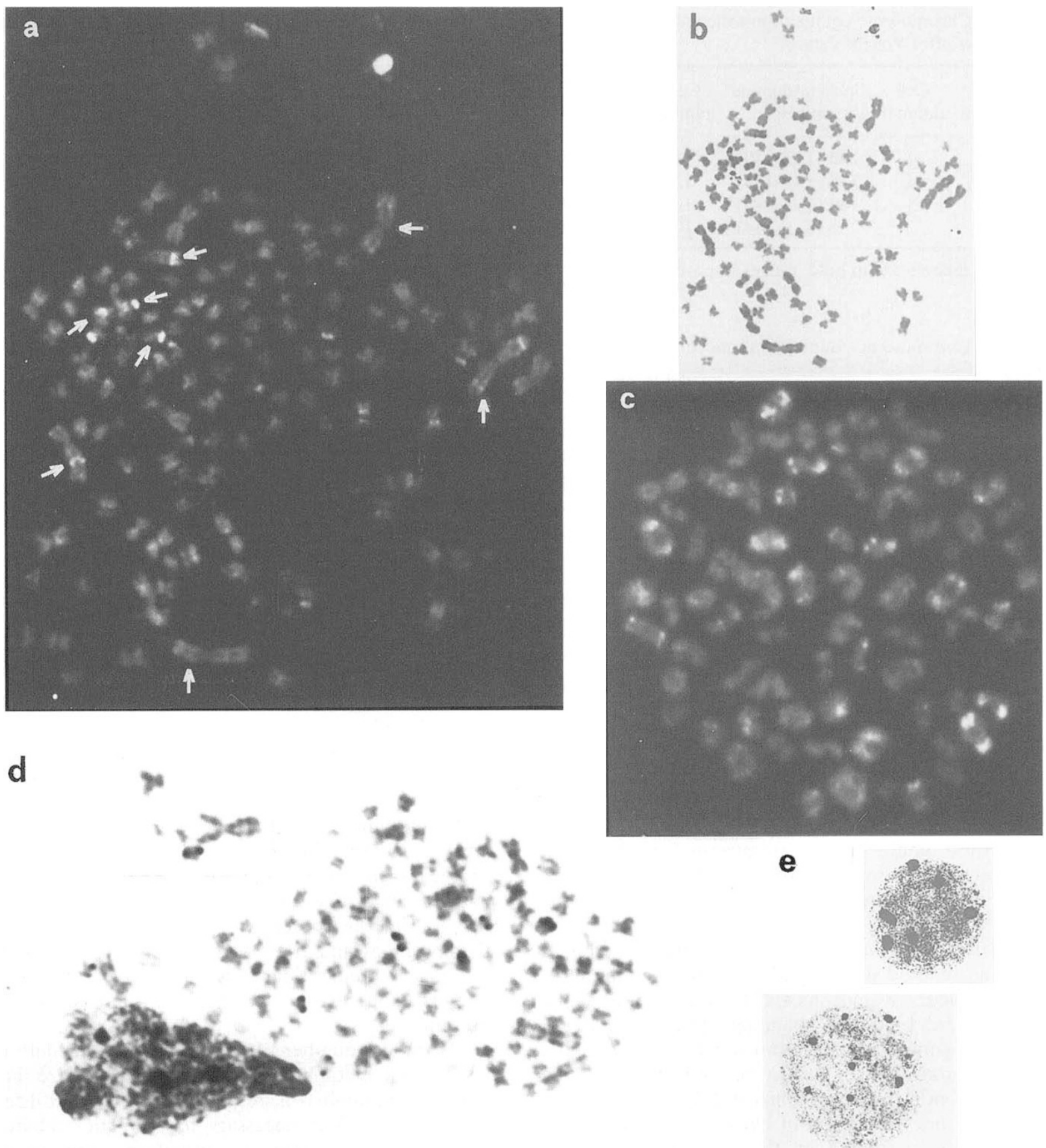

Fig. 4 (a) Mitotic embryonal metaphase after quinacrine staining: bright bands are localized on the short arms of some small submetacentric chromosomes (arrowed) and a few large chromosomes show intercalary and subtelomeric bands in both arms (arrowed). (b) The same metaphase plate after Wright's stain. (c) Meiotic metaphase I after quinacrine staining: a few bivalents with AT-rich clusters are easily identifiable. In this phase, B chromosomes are difficult to identify because of their tight condensation. (d) NOR silver staining: five to eight Ag-positive regions are identifiable; they are localized on the short arm of small telocentric chromosomes as well as on the centromeric region of a small metacentric chromosome pair. The chromosomes top left have been included in an insert because, although belonging to the same metaphase plate, they were out of the field. (e) Interkinetic nuclei of the embryos showing six and eight nucleoli.

(c) The Genetical Society of Great Britain, Heredity, 76, 92-99. 
Table 1 Chromosome counts of mitotic and meiotic configurations of Nephrops norvegicus after Wright's stain

\begin{tabular}{lcrrrrr}
\hline Specimen & $\begin{array}{c}\text { Cell } \\
\text { number }\end{array}$ & $\begin{array}{c}\text { Spermatogonial } \\
\text { mitosis }\end{array}$ & $\begin{array}{c}\text { Cell } \\
\text { number }\end{array}$ & $\begin{array}{c}\text { Metaphase } \\
\text { I }\end{array}$ & $\begin{array}{c}\text { Cell } \\
\text { number }\end{array}$ & $\begin{array}{c}\text { Metaphase } \\
\text { II }\end{array}$ \\
\hline I & 4 & $\mathbf{1 4 0}(132-140)$ & 12 & $\mathbf{7 4}(72-75)$ & 12 & $\mathbf{7 0}(68-72)$ \\
II & 4 & $\mathbf{1 3 6}(134-138)$ & 12 & $\mathbf{7 2}(69-74)$ & 26 & $\mathbf{6 9}(65-73)$ \\
III & 3 & $\mathbf{1 4 0}(138-140)$ & 10 & $\mathbf{7 2}(70-74)$ & 11 & $\mathbf{6 8}(68-70)$ \\
IV & 2 & $(133-134)$ & 10 & $\mathbf{7 3}(70-74)$ & 7 & $\mathbf{6 8}(64-72)$ \\
\hline
\end{tabular}

Modal numbers are in bold, ranges in parentheses.

Table 2 Chromosome counts of mitotic and meiotic configurations of Nephrops norvegicus after C- and DdeI-induced banding

\begin{tabular}{|c|c|c|c|c|c|c|c|c|}
\hline \multirow{3}{*}{$\begin{array}{c}\text { Cell } \\
\text { number }\end{array}$} & \multicolumn{2}{|c|}{ Spermatogonial mitosis } & \multirow{3}{*}{$\begin{array}{c}\text { Cell } \\
\text { number }\end{array}$} & \multicolumn{2}{|c|}{ Metaphase I } & \multirow{3}{*}{$\begin{array}{c}\text { Cell } \\
\text { number }\end{array}$} & \multicolumn{2}{|c|}{ Metaphase II } \\
\hline & A & B & & & B & & A & B \\
\hline & & & & Bivalents & chromos & & chromosomes & chromoss \\
\hline 6 & $123(123-132)$ & $8(7-8)$ & 12 & $66(65-66)$ & $8(8-9)$ & 9 & $65(65-67)$ & $4(3-5)$ \\
\hline 6 & $125(124-130)$ & $8(7-9)$ & 15 & $64(63-66)$ & $8(6-9)$ & 8 & $65(64-66)$ & $4(3-5)$ \\
\hline 3 & $132(131-132)$ & $8(8-9)$ & 5 & $64(63-64)$ & $8(7-9)$ & 10 & $64(63-66)$ & $4(4-5)$ \\
\hline
\end{tabular}

Modal numbers are in bold, ranges in parentheses.

\section{Discussion}

Karyological studies on Decapoda have so far supplied general information on the karyotype structure, morphology and behaviour of chromosomes (Niiyama, 1959; Nakamura, 1988). Difficulties relating to the nature of the chromosomes of Decapoda and technical constraints of the methodologies used for cytological preparations have not allowed any further progress.

In this study we show that a routine metaphase staining method does not allow correct counting of $N$. norvegicus chromosomes. C-banding as well as the similar DdeI-induced banding (Sumner, 1990), differentiating some chromosomal regions and whole chromosomes, gave a better insight into the nature of numerical variability in this species. Moreover, the fine resolution of chromosomes in mitosis and meiosis obtained by the enzyme digestion is useful in understanding and identifying B chromosomes. These chromosomes are generally heterochromatic and unaffected by DdeI, asynaptic or disjoined early in metaphase I and present a distorted meiotic segregation, clearly shown by metaphases II from the same meiocyte (Figs 2 and 3). In metaphase I they are also tightly condensed and their primary constriction is often difficult to identify to the extent that they are indistinguishable from bivalents after conventional staining. Therefore, a count of bivalents in these conditions leads to an overassessment of the chromosome number (Table 1).

By counting $\mathrm{C}+$ and $D d e \mathrm{I}+$ chromosomes separately from the other members of the complement (Table 2), variability is greatly reduced. However, a narrow range of variation is still present. This may result from the presence of nonheterochromatic $B$ chromosomes and/or from the normal variability of cytological preparations when a high chromosome number and extremely small chromosomes are present.

The modal number of $\mathrm{B}$ chromosomes in mitotic and meiotic metaphases leads us to hypothesize that a minimum number of, respectively, seven and three B chromosomes is necessary for gametic viability. Distorted segregation is therefore functionally limited and a control mechanism could be hypothesized.

The B chromosomes of the first and the second group in $N$. norvegicus are also peculiar for their unusually large size. Indeed they include the largest members of the complement whereas in most species they are smaller than or equal to the normal A chromosome complement. Large B chromosomes

(c) The Genetical Society of Great Britain, Heredity, 76, 92-99. 
are present in a few species (Jones \& Rees, 1982) and recently have been found in some species of tropical fishes (Maistro, 1992).

The particular information in this study relates to the presence and identification of some supernumerary chromsomes. Nephrops norvegicus shows a very low genetic variability (Mantovani \& Scali, 1992). B chromosome polymorphism could be a useful marker for the investigation of the possible variability among allopatric populations.

\section{Acknowledgements}

We thank Professor A. Cau, U. Laudani and A. Marchi for helpful advice during the development of this research and Professor R. N. Jones for critical reading of the manuscript.

\section{References}

CASPERRSON, T., ZECH, L., MODEST, E. J., FOLEY, G. E., WAGH, U. AND SIMONSSON, E. 1969. Chemical differentiation with fluorescent alkylating agents in Vicia faba metaphase chromosomes. Exp. Cell Res., 58, 128-140.

CAU, A., SAlvadori. S., Deiana, A. M., Bella, I. L. AND MEZZANOTTE, R. 1988. The characterization of Muraena helena L. mitotic chromosomes: karyotype, C-banding, nucleolar organizer regions and in situ digestion with restriction endonucleases. Cytogenet. Cell Genet., 47, 223-226.

CORNI, M. G., TRENTINI, M. AND FRoGliA, C. 1989. Karyological study on Nephrops norvegicus (L., 1758) (Astacidea, Nephropidae) in the Central Adriatic sea. Nova Thalassia, 10, 127-131.

DOUSSAU DE BAZIGNAN, M. AND OZOUF-COSTAZ, c. 1985. Une technique rapide d'analyse chromosomique appliquée à sept espèces de poissons antarctiques. Cybium, 9, 57-74

FARMER, A. S. 1974. A new technique applied to the chromosomes of Nephrops norvegicus (L.) (DECAPODA, NEPHROPIDAE). Crustaceana, 27, 17-21.

HuGHES, J. B. 1982. Variability of chromosome number in the lobsters, Homarus americanus and Homarus gammarus. Caryologia, 35, 279-289.

JONES, R. N. 1985. Are B-chromosomes selfish? In: Cavalier-Smith, T. (ed.) The Evolution of Genome Size, pp. 397-425. John Wiley, London.

JONES, R. N. AND REES, H. 1982a. Structure and organization. In: Jones, R. N. and Rees, H. (eds) B Chromosomes, pp. 19-39. Academic Press, New York.

JONES. R. N. AND REES, H. 1982b. Transmission and inheritance. In: Jones, R. N. and Rees, H. (eds) B Chromosomes, pp. 40-68. Academic Press, New York.

KLINKHARDT, M. 1992. Direct methods for chromosome preparations from embryos. In: Ozouf-Costaz, C. (ed.) First International Workshop on Fish Cytogenetic Techniques, pp. 35-37. Concarneau, France.

MAISTRO, E. L., FORESTI, F., OLIVEIRA, C. AND ALMEIDATOLEDO, L. F. 1992. Occurrence of macro B chromosomes in Astyanax scabripinnis paranae (Pisces, Characiformes, Characidae). Genetica, 87, 101-106.

MANTOVANI, B. AND SCALl, V. 1992. Allozyme characterization of the Norway lobster, Nephrops norvegicus, of two Adriatic trawling grounds. Acta Adriat., 33, 209-213.

NAKAMURA, H. K., MACHII, A., WADA, K. T., AWAJI, M. AND TOWNSLEY, S. J. 1988. A check list of Decapod chromosomes (Crustacea). Bull. Natl. Res. Inst. Aquaculture, 13, $1-9$.

NIIYAMA, H. 1959. A comparative study of the chromosomes in Decapods, Isopods and Amphipods, with some remarks on cytotaxonomy and sex-determination in the Crustacea. Mem. Fac. Fish. Hokkaido Univ., 7, $1-60$.

ROBERTS, F. L. 1969. Possible supernumerary chromosomes in the Lobster, Homanus americanus. Crustaceana, 16, 194-196.

SUMNER, A. T. 1990. Banding with nucleases. In: Sumner, A. T. (ed.) Chromosome Banding, pp. 259-265. Unwin Hyman, London. 